\title{
Gorensteinness and iteration of Cox rings for Fano type varieties
}

\author{
Lukas Braun ${ }^{1}$
}

Received: 22 September 2020 / Accepted: 2 December 2021 / Published online: 9 January 2022

(c) The Author(s) 2022

\begin{abstract}
We show that finitely generated Cox rings are Gorenstein. This leads to a refined characterization of varieties of Fano type: they are exactly those projective varieties with Gorenstein canonical quasicone Cox ring. We then show that for varieties of Fano type and Kawamata $\log$ terminal quasicones $X$, iteration of Cox rings is finite with factorial master Cox ring. In particular, even if the class group has torsion, we can express such $X$ as quotients of a factorial canonical quasicone by a solvable reductive group.
\end{abstract}

Keywords Fano varieties · Quasicones · Mori Dream spaces · Gorenstein · Iteration of Cox rings

Mathematics Subject Classification 14J45 $\cdot 14 \mathrm{M} 05 \cdot 13 \mathrm{~A} 02$

\section{Contents}

Introduction . . . . . . . . . . . . . . . . . . . . . . . . . . . . 1047

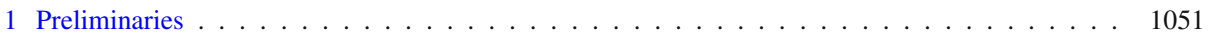

2 Almost principal quasitorus bundles . . . . . . . . . . . . . . . . . . . . . . . 1051

3 Cox rings are Gorenstein . . . . . . . . . . . . . . . . . . . . . . . . . . . . . 1054

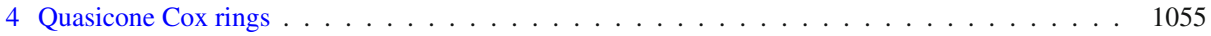

5 Iteration of Cox rings . . . . . . . . . . . . . . . . . . . . . . 1056

References . . . . . . . . . . . . . . . . . . . . . . . . 1059

\section{Introduction}

Let $X$ be a normal algebraic variety over the field $\mathbb{C}$ of complex numbers. Assume that $X$ has only constant global invertible regular functiones, e.g. $X$ is projective. If $X$ has finitely generated divisor class group $\mathrm{Cl}(X)$, one can define its $\mathrm{Cl}(X)$-graded Cox ring

Lukas Braun

lukas.braun@math.uni-freiburg.de

1 Mathematisches Institut, Albert-Ludwigs-Universität Freiburg, Ernst-Zermelo-Strasse 1,

79104 Freiburg im Breisgau, Germany 


$$
\mathcal{R}(X):=\bigoplus_{\mathrm{Cl}(X)} \Gamma\left(X, \mathcal{O}_{X}(D)\right)
$$

and ask if it is a finitely generated $\mathbb{C}$-algebra. If so, $X$ is called a Mori Dream Space (MDS) due to $\mathrm{Hu}$ and Keel [29], who showed that this property is equivalent to a good behaviour with respect to the minimal model program, whence the name.

Note that this definition differs from the original one of $\mathrm{Hu}$ and Keel, as we do not assume $X$ to be projective and $\mathbb{Q}$-factorial, and in that $\mathcal{R}(X)$ is graded by $\mathrm{Cl}(X)$, not $\operatorname{Pic}(X)$. Moreover, some care has to be taken if $\mathrm{Cl}(X)$ has torsion. The original reference for this case is [9], where also the case of non-finitely generated $\mathcal{R}(X)$ is investigated. See also the comprehensive work [5].

Examples of MDS include toric [14] and more general spherical [12] varieties as well as certain ones with an action of a torus of lower dimension, see [26]. It was shown in [10] that varieties of Fano type - i.e. projective varieties admitting an effective $\mathbb{Q}$-divisor $\Delta$ such that $-\left(K_{X}+\Delta\right)$ is ample and $(X, \Delta)$ is Kawamata log terminal (klt) - are MDS.

Cox rings of MDS enjoy many nice properties: The $\mathrm{Cl}(X)$-grading induces an action of the characteristic quasitorus $H_{X}:=\operatorname{Spec} \mathbb{C}[\mathrm{Cl}(X)]$ on the total coordinate space $\bar{X}:=$ $\operatorname{Spec} \mathcal{R}(X)$, and there is an open subvariety $\widehat{X} \subseteq \bar{X}$ with complement of codimension at least two such that $X$ is isomorphic to the good quotient $\widehat{X} / / H_{X}$. We call $\widehat{X}$ the characteristic space.

In [9] and with different methods in [3,17] it was shown that if $\mathrm{Cl}(X)$ is free, then $\mathcal{R}(X)$ is factorial and thus in particular it is Gorenstein, i.e. the canonical class $K_{\bar{X}}$ is $\operatorname{Cartier}$. If $\mathrm{Cl}(X)$ is not free, then $\mathcal{R}(X)$ is still factorially graded in the sense that homogeneous elements can be expressed as a unique product of irreducible homogeneous ones, see [3], but properties such as Gorensteinness do not follow from this. Moreover, it was shown independently in $[13,20,30]$ that a projective variety $X$ is of Fano type if and only if $\mathcal{R}(X)$ is log terminal. Analogously, (projective) MDS are shown to be of Calabi-Yau type if and only if $\mathcal{R}(X)$ is $\log$ canonical [20,30]. In [13], Brown also observed that if $X$ is smooth and Fano, then $\mathcal{R}(X)$ is Gorenstein canonical. Our first result is the following:

Theorem 1 Let $X$ be an MDS. Then $\mathcal{R}(X)$ is Gorenstein.

As Brown points out [13], a finitely generated Cox ring need not be Cohen Macaulay. But since $K_{\bar{X}}$ is Cartier, if e.g. $\bar{X}$ has rational singularities, it is not only Cohen Macaulay, but even canonical.

Recall that a quasicone is an affine variety with a $\mathbb{C}^{*}$-action such that all closures of $\mathbb{C}^{*}$ orbits meet in one common point, the vertex. We will call the coordinate ring of such affine variety a quasicone as well. In fact, for an MDS that is either complete or itself a quasicone, $\bar{X}$ is a quasicone, cf. Proposition 3 .

By [35, Le. 5.1], quasicones $X$ have trivial Picard group (normality is essential here, see Remark 5), yet the whole class group is generated by the local class group at the vertex and $K_{X}$ is even trivial if $X$ is Gorenstein. In fact, klt quasicones turn out to be the affine counterparts of Fano type varieties, so we can refine the characterization from $[13,20,30]$ :

Theorem 2 Let $X$ be projective (affine). Then it is of Fano type (a klt quasicone) if and only if $\bar{X}$ is a Gorenstein canonical quasicone.

Note that the property 'Fano type / klt quasicone' does not only guarantee MDS-ness, but is also preserved when passing to the total coordinate space $\bar{X}$. Thus it is natural to consider the Cox ring and total coordinate space $X^{(2)}:=\overline{\bar{X}}$ of $X^{(1)}:=\bar{X}$, then the ones of $X^{(2)}$ etc., we call this the iteration of Cox rings. 
Iteration of Cox rings has been introduced by Arzhantsev, Hausen, Wrobel and the author in [4] for affine rational varieties with a torus action of complexity one. In general, for an MDS $X$, three scenarios are possible: either $X$ has infinite iteration of Cox rings, or at some point $X^{(N)}$, the iteration stops. The latter may have two reasons: either $X^{(N)}$ is non-MDS or it is factorial, i.e. its own total coordinate space. We call $\mathbb{C}\left[X^{(N)}\right]$ the master Cox ring of $X$.

It was shown in [4] that log terminal quasicones with a torus action of complexity one have finite iteration of Cox rings with factorial master Cox ring. In [27], Hausen and Wrobel determined which non-quasicone affine rational varieties with a torus action of complexity one have finite iteration with factorial master Cox ring. In fact, it can be deduced from their observations that all rational varieties with a torus action of complexity one have finite iteration of Cox rings, though with possibly non-MDS master Cox ring. Toric varietiesin a trivial way, since $\mathcal{R}(X)$ is a polynomial ring - and also spherical varieties - see [19, Thm. 1.1]— have factorial master Cox ring. We prove the following:

Theorem 3 Let $X$ be of Fano type or a klt quasicone. Then $X$ has finite iteration of Cox rings with factorial master Cox ring.

Note that for any $i \leq N$, the quasitorus quotients in the iteration of Cox rings can be combined to express $X$ as a GIT-quotient $X^{(i)^{\text {ss }}} / / G$ of $X^{(i)}$ by a solvable group $G$, see Proposition 4. In the case of $\log$ terminal surface singularities, this yields the presentation as finite quotients of $\mathbb{C}^{2}$ and, in addition, the derived normal series of the respective finite groups, see [4]. Note that our observations till now guarantee MDS-ness of all $X^{(i)}$ in the iteration of Cox rings for klt quasicones and thus Fano type varieties, but they do not yield finiteness of the iteration. To prove this, we use a theorem of Greb, Kebekus and Peternell [21, Thm. 1.1], stating (roughly) that in a sequence of finite morphisms between klt spaces that are étale over smooth loci all but finitely many must be étale.

In order to apply this result, we need to pass from in general nonfinite quasitorus quotients to finite Galois covers. This is made possible by the observation made in Corollary 1, which we state here as Lemma 1 as it is essential for most of our statements, including Theorem 1 on Gorensteinness of the Cox ring. For convenience, we will mostly depict torus quotients by vertical, finite quotients by horizontal and quasitorus quotients by diagonal arrows.

Lemma 1 Let $X$ be an MDS and $H_{X}=E \times \mathbb{T}$ its characteristic quasitorus with torsion and torus part $E$ and $\mathbb{T}$ respectively. Then:

(i) The Cox rings of $X$ and of the geometric quotient $X_{E}:=\widehat{X} / E$ coincide. In particular, $X_{E}$ is a quasiaffine MDS and almost factorial.

(ii) The characteristic space $\widehat{X}$ is an MDS if and only if $X_{\mathbb{T}}:=\widehat{X} / / \mathbb{T}$ is an MDS and in that case, their Cox rings coincide.

In particular, we have the following commutative diagram of quasitorus quotients, where CR denotes characteristic spaces:

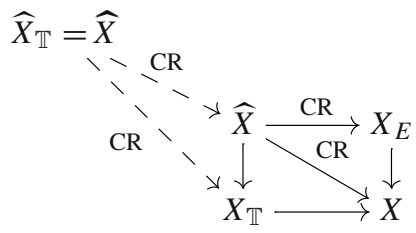

Here, (i) is essential in the proof of Theorem 1 as it allows to reduce to the case of finite $\mathrm{Cl}(X)$ while (ii) allows to pass from quasitorus quotients to finite Galois covers in the proof of 
Theorem 3. The first main ingredience of Lemma 1 is a theorem of Bechtold [8, Thm. 1.5], stating that factoriality of a grading (which is essential for Cox rings) is preserved when changing the free part of the grading group. The second underlying observation is that if an MDS $X$ has a quotient presentation $X=Y / / H$ with a variety $Y$ and a quasitorus $H$ with certain good properties, see [5, Def. 4.2.1.1], then the presentation $\widehat{X} \rightarrow X$ factors through $Y \rightarrow X$, see [5, Thm. 4.2.1.4]. In analogy to [5], the term quotient presentation will be used only for abelian quotients in the following.

In view of this, consider Okawa's Theorem [36, Thm. 1.1], which states that for a surjective morphism $f: X \rightarrow Y$ between projective varieties, if $X$ is an MDS then $Y$ is as well. We can in fact say more if $f$ is a (quasitorus) quotient presentation, namely if $Y$ and $\widehat{Y}$ are MDS, then $X$ is so, see Proposition 1. In terms of iteration of Cox rings, this generalizes to the following:

Theorem 4 Let $\cdots \rightarrow X_{4} \rightarrow X_{3} \rightarrow X_{2} \rightarrow X_{1}$ be a chain of quotient presentations. Denote the $i$-th iterated characteristic space of $X_{j}$ by $X_{j}^{(i)}$ if it exists, i.e. $X_{j}^{(1)}:=\widehat{X}_{j}$ etc. Then if one of the $X_{i}$ has infinite iteration of Cox rings, the others have as well. If one has factorial master Cox ring, the others have as well and in that case, all master Cox rings coincide. In these cases, we get a web of Cox ring iterations:

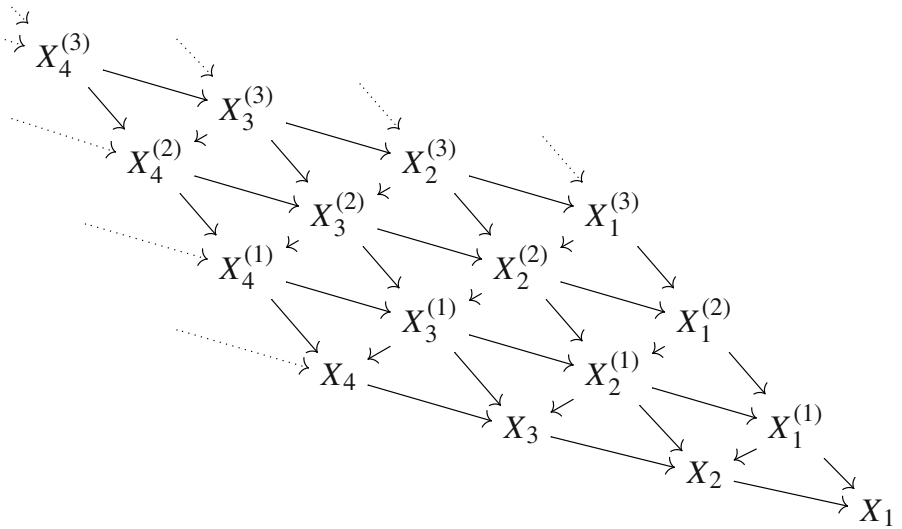

Note that if for example $X_{1}$ has finite iteration of Cox rings with non-MDS master Cox ring $\mathbb{C}\left[X_{1}^{(3)}\right]$, we get MDS-ness only for $X_{2}, X_{2}^{(1)}, X_{3}$. So in such case, the maximal number of steps in the Cox ring iteration becomes important.

Due to [5, Thm. 4.2.1.4], the characteristic space $\widehat{X} \rightarrow X$ is a universal reductive abelian (possibly nondiscrete) 'cover' of $X$ in the sense that it factors through any quotient presentation $Y \rightarrow X$. This can be generalized for MDS with finite iteration of Cox rings and factorial master Cox ring in the following way.

Corollary 1 Let $X$ be an MDS with finite iteration of Cox rings and factorial master Cox ring. Let also $X=Y / / G$ be a quotient of a normal variety $Y$ by a solvable reductive group $G$, such that $Y$ has only constant invertible $G$-invariant functions and $G$ acts freely on a subset with complement of codimension at least two in $Y$. Then the quotient $\left(X^{(N)}\right)^{\mathrm{ss}} \rightarrow X$, where $\left(X^{(N)}\right)^{\mathrm{ss}}$ denotes the set of semistable points in $X^{(N)}$, factors through $Y \rightarrow X$. We call $\left(X^{(N)}\right)^{\mathrm{ss}} \rightarrow X$ the universal solvable quotient presentation of $X$. 
Note that this does not mean in general that a solvable quotient $Y \rightarrow X$ factors through $\widehat{X} \rightarrow X$, compare [6, §3] for the affine case. Moreover, if $X$ has non-MDS master Cox ring, difficulties as with Theorem 4 arise.

\section{Preliminaries}

In this section, we shortly discuss notions and definitions relevant for the article, where for a detailed discussion we refer to $[5,9,29,34]$. Further definitions are given directly in the respective sections.

Notions for singularities of pairs are standard, we refer to [32]. A variety of Fano type is a projective variety $X$, such that there exists an effective $\mathbb{Q}$-divisor $\Delta$ with $(X, \Delta)$ klt and $-\left(K_{X}+\Delta\right)$ ample.

We use the following notions concerning the Picard and divisor class group: if $\mathrm{Cl}(X)$ is trivial (torsion), we say $X$ is factorial (almost factorial). If the group $\mathrm{Cl}(X) / \mathrm{Pic}(X)$ is trivial (torsion), we say that $X$ is locally factorial $(\mathbb{Q}$-factorial).

Our object of interest are Mori Dream Spaces (MDS), which for us will be normal algebraic varieties with a finitely generated divisor class group and finitely generated Cox ring

$$
\mathcal{R}(X):=\bigoplus_{\mathrm{Cl}(X)} \Gamma\left(X, \mathcal{O}_{X}(D)\right) .
$$

The original definition [29, Def. 1.10] of $\mathrm{Hu}$ and Keel was in terms of a good behaviour with respect to the minimal model program, but they showed in [29, Prop. 2.9] that this is equivalent to a finitely generated Picard group $\operatorname{Pic}(X)$ and $\operatorname{Pic}(X)$-graded Cox ring at least for $\mathbb{Q}$-factorial projective varieties.

These definitions have been generalized to not necessarily separated prevarieties and $\mathrm{Cl}(X)$-graded Cox rings [5,25]. Sometimes only the free part of $\mathrm{Cl}(X)$ is taken, but the differences are then limited to finite abelian covers, see [20, Ch. 2, 5]. Finite generation of $\operatorname{Pic}(X)$ and $\mathrm{Cl}(X)$ is equivalent for $\mathbb{Q}$-factorial varieties and in the non- $\mathbb{Q}$-factorial case at least for klt pairs $[10$, Cor. 1.4.3]. Non- $\mathbb{Q}$-factorial MDS always have a small $\mathbb{Q}$-factorial birational modification [2, Thm. 2.3].

For technical reasons (at least in the case when $\mathrm{Cl}(X)$ has torsion), we always assume $\Gamma\left(X, \mathcal{O}^{*}\right)=\mathbb{C}^{*}$, see [5, Sec. 1.4.2]. Note that for complete varieties and quasicones, this condition is satisfied and preserved by the Cox ring, which is not the case in general, see [5, Ex. 1.4.4.2].

\section{Almost principal quasitorus bundles}

In [24], Hashimoto introduced the notion of almost principal fiber bundles. The definition is as follows:

Definition 1 Let $Y$ and $X$ be normal varieties, moreover let $G$ be an affine algebraic group, acting on $Y$ such that $\varphi: Y \rightarrow X=: Y / / G$ is a good quotient. Then we call $\varphi$ an almost principal $G$-bundle if there exist open subsets $U \subseteq Y$ and $V \subseteq X$ with complement of codimension at least two so that

$$
\left.\varphi\right|_{U}: U \rightarrow V
$$

is a principal $G$-bundle. 
This notion naturally comes into play in the setting of Cox rings, as the representation of an MDS $X$ as the quotient $\widehat{X} / / H_{X}$ is of such kind, see [5, Prop. 1.6.1.6]. In fact, $V$ can always be chosen to be the regular locus of $X$. Moreover, the quotient presentations from [5, Def. 4.2.1.1] having the property that the Cox construction factors through them are almost principal quasitorus bundles with the additional assumption that all invertible functions homogeneous with respect to the grading group are constant. We will in the following use the notions quotient presentation and almost principal quasitorus bundle interchangeably, where we assume only constant invertible homogeneous functions and that $V$ from Definition 1 can be chosen as $X_{\text {reg }}$.

Okawa has shown in [36] that if $f: X \rightarrow Y$ is a surjective morphism of projective varieties and $X$ is an MDS, then $Y$ is as well. Bäker showed in [7] that the same holds if $f: X \rightarrow Y=X / / G$ is a quotient presentation with not necessarily projective normal $X$ and a reductive affine algebraic group $G$. We can in fact say more if $G$ is a quasitorus:

Proposition 1 Let $X$ and $Y$ be normal algebraic varieties with constant global invertible regular functions. Let $\varphi: X \rightarrow Y$ be an almost principal $H$-bundle of normal varieties with $H$ a quasitorus. We have the following:

(i) If $H$ is a torus, then $X$ is an MDS if and only if $Y$ is so and in this case $\mathcal{R}(X)=\mathcal{R}(Y)$.

(ii) If $X$ is an MDS, then $Y$ is so and we have a commutative diagram of almost principal quasitorus bundles:

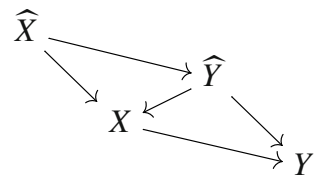

(iii) If $Y$ is an MDS, then its characteristic space is an almost principal quasitorus bundle over $X$, i.e. we have at least the following commutative subdiagram:

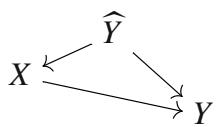

Moreover, if $\widehat{Y}$ is an MDS, then $X$ is so and we get the enhanced diagram

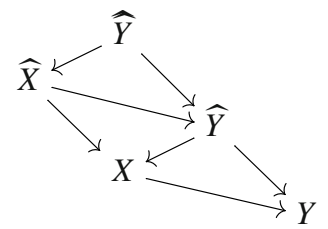

Remark 1 One can observe that here iteration of Cox rings comes into play, that is, if $Y$ has iteration of Cox rings with at least two steps, then $X$ is an MDS. For further generalizations see Proposition 5 and of course Theorem 4.

Before proving Proposition 1, we state a corollary that is crucial for both Gorensteinness of Cox rings and finiteness of Cox ring iteration. It shows that among the quotient presentations of $X$, the two exceptional ones $\widehat{X} / E \rightarrow X$ and $\widehat{X} / / \mathbb{T} \rightarrow X$-with $E$ and $\mathbb{T}$ being the torsion and torus part of $H_{X}$ - have very special properties. 
Corollary 1 (Lemma 1) Let $X$ be an MDS and $H_{X}=E \times \mathbb{T}$ its characteristic quasitorus with torsion and torus part $E$ and $\mathbb{T}$ respectively. We have the following:

(i) The Cox rings of $X$ and of the geometric quotient $X_{E}:=\widehat{X} / E$ coincide. In particular, $X_{E}$ is a quasiaffine MDS and almost factorial.

(ii) The characteristic space $\widehat{X}$ is an MDS if and only if $X_{\mathbb{T}}:=\widehat{X} / / \mathbb{T}$ is an MDS and in that case, their Cox rings coincide.

Proof The assertions follow directly if one of $E$ or $\mathbb{T}$ is trivial-note that $\widehat{X}$ is factorial in the first case. So assume that none of them is trivial.

We begin with (i). By Proposition 1, since $X_{E} \rightarrow X$ is an almost principal torus bundle, both share the same Cox ring. Thus $E$ is the characteristic quasitorus of $X_{E}$ and since it is finite and $X_{E}$ is open in $\bar{X} / E$, which is affine, it is quasiaffine and almost factorial. Also (ii) follows directly from (i) of Proposition 1.

Remark 2 In fact in Corollary 1 (i), we can replace $E$ by $E \times T$ for any subtorus $T \subseteq \mathbb{T}$ and still $X_{E \times T}$ has Cox ring $\mathcal{R}(X)$, while quasiaffineness may fail.

Proof (Proof of Proposition 1) We consider the first assertion. If $X$ is an $\mathrm{MDS}$, then $\mathrm{Cl}(X)$ is finitely generated and $\mathcal{R}(X)$ is factorially $\mathrm{Cl}(X)$-graded. Thus by [8, Thm. 1.5], it is also factorially $\mathrm{Cl}(X) \times \mathbb{X}(H)$-graded, since the character group $\mathbb{X}(H)$ of $H$ is torsion free. By [7, Le. 3.1], the class group $\mathrm{Cl}(Y)$ is finitely generated. We note that the lifting of $H$ to $\widehat{X}$, c.f. [5, Thm. 4.2.3.2], provides a GIT-quotient $\widehat{X} \rightarrow Y$ for the $H_{X} \times H$-action on $\bar{X}$ coming from the $\mathrm{Cl}(X) \times \mathbb{X}(H)$-grading. Since $X \rightarrow Y$ is a quotient presentation, by [5, Thm. 4.2.1.4], it corresponds to a subgroup of $\mathrm{Cl}(Y)$ and in particular is a locally trivial $H$-bundle over $Y_{\text {reg }}$, with the preimage of $Y_{\text {reg }}$ lying in $X_{\text {reg }}$. As a characteristic space, $\widehat{X} \rightarrow X$ is a locally trivial $H_{X}$-bundle over $X_{\text {reg. }}$. Thus we see that $\widehat{X} \rightarrow Y$ is a locally trivial $H_{X} \times H$-bundle over $Y_{\text {reg }}$. By [5, Thm. 1.6.4.3], we get that $\widehat{X} \rightarrow Y$ is a characteristic space for $Y$. In particular, $Y$ is an MDS and its Cox ring coincides with $\mathcal{R}(X)$.

Now assume $Y$ is an MDS. Then by [5, Thm. 4.2.1.4], we have a commutative diagram of quotient presentations

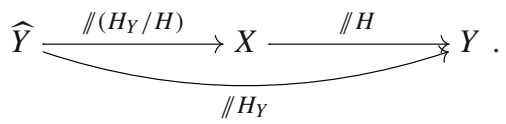

Since $\mathcal{R}(Y)$ is factorially $\mathrm{Cl}(Y)$-graded and $\mathrm{Cl}(Y)=\mathbb{X}\left(H_{Y} / H\right) \times \mathbb{X}(H)$, it is factorially $\mathbb{X}\left(H_{Y} / H\right)$-graded by [8, Thm. 1.5]. By [5, Thm. 1.6.4.3], the Cox ring of $X$ is $\mathcal{R}(Y)$.

We come to assertion (ii). If $X$ is an MDS, then $Y$ is so by [7, Thm. 1.1] and by [5, Thm. 4.2.1.4], we get a quotient presentation $\widehat{Y} \rightarrow X$. Applying [5, Thm. 4.2.1.4] again, we get a quotient presentation $\widehat{X} \rightarrow \widehat{Y}$ and thus the desired diagram. For (iii), apply [5, Thm. 4.2.1.4] several times as above.

The following proposition uses reduction to positive characteristic. We refer to $[23,28,40]$ for definitions and details, with an emphasis on Cox ring related problems in $[1,20]$.

The main purpose of [24] is to show how properties are preserved by almost principal fiber bundles. Varying the finite version [32, Prop. 5.20], we show the following on preservation of log-terminality by almost principal fiber bundles.

Proposition 2 Let $\varphi: X \rightarrow Y:=X / / G$ be an almost principal $G$-bundle with $G$ an affine algebraic group. Let $X$ and $Y$ be $\mathbb{Q}$-Gorenstein. Then $X$ is log-terminal if and only if $Y$ is so. 
Proof By [41, Cor. 3.4], a pair $\left(Z, \Delta_{Z}\right)$ is klt if and only if it is of locally $F$-regular type in the sense that all local rings are of strongly $F$-regular type. So if $X$ is $\mathbb{Q}$-Gorenstein, the pair $(X, 0)$ is of locally $F$-regular type. Then by [24, Thm. 13.14], $Y$ allows a divisor $\Delta$, such that the pair $(Y, \Delta)$ is klt. Since $Y$ is $\mathbb{Q}$-Gorenstein, it is log-terminal. The other direction is analogous.

Remark 3 If [24, Thm. 13.14] is valid for pairs as well, then we can state Proposition 2 also for pairs $\left(X, \Delta_{X}\right),\left(Y, \Delta_{Y}\right)$. Also note that there is a different definition of being of locally $F$-regular type than the one used above. Namely the a priori stronger one that $Z$ is covered by affine globally $F$-regular subsets, see [40, Def. 3.1]. One can show that these two notions are in fact equivalent, see [39] with slightly different definitions.

The statement of Proposition 2 has a different, more local nature than the corresponding ones [20, Prop. 4.6] and [1, Prop. 7.17]. These guarantee not only log-terminality but the global property of being of Fano type for a variety $X$ if the Cox ring is log-terminal. Combining these two viewpoints, we get the following.

Corollary 2 Let $X$ be a log-terminal MDS that is not of Fano type. Then the complement of $\widehat{X}$ in $\bar{X}$ contains all non log-terminal singularities of $\bar{X}$.

\section{Cox rings are Gorenstein}

The purpose of this section is to prove Theorem 1. For convenience, we state it here again in the following form:

Theorem 1 (Theorem 1) Let $X$ be an MDS. Then $\mathcal{R}(X)$ is a numerically Gorenstein ring. In particular, if $\bar{X}$ is Cohen Macaulay, it is Gorenstein. If $\bar{X}$ is rational, it is Gorenstein canonical.

Remark 4 In general, one cannot expect a finitely generated Cox ring to be Cohen Macaulay or $\mathbb{Q}$-factorial. For a non Cohen Macaulay example by Gongyo, see the paper [13] by Brown. For a collection of non $\mathbb{Q}$-factorial examples, see [4, Thm. 1.9], where the toric one given by $x y+z^{a} w^{b}$ is probably the easiest one. It is the Cox ring of the affine threefold with $\left(\mathbb{C}^{*}\right)^{2}$-action given by

$$
x^{2}+y^{2} z+z^{a} w^{b} .
$$

Proof (Proof of Theorem 1) If $H_{X}$ is torsion free, then $\bar{X}$ is factorial and thus numerically Gorenstein. Otherwise, consider $Y:=\widehat{X} / E$, where $E$ is the torsion part of $H_{X}$. Then $Y$ is almost factorial by Corollary 1 . In fact, every Weil divisor is $\mathbb{Q}$-principal. Then a multiple of $K_{X}$ is principal, say $r K_{X}=0$ in $\mathrm{Cl}(Y)$. This means we have its global canonical or index-1cover $\rho: \mathcal{Y} \rightarrow Y$, see [32, 5.19]. Since $\rho$ is étale over $Y_{\text {reg }}, \mathbb{Z} / r \mathbb{Z}$ acts strongly stably on $\mathcal{Y}$ so that $\rho$ becomes a quotient presentation of $Y$. Now [5, Thm. 4.2.1.4] gives us the commutative diagram of quotient presentations

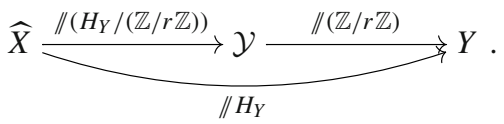

Note that even if $\mathcal{R}(\mathcal{Y})$ exists, it may differ from $\mathcal{R}(X)$. Now since $K_{\mathcal{Y}}$ is Cartier and $\widehat{X} \rightarrow \mathcal{Y}$ is unramified over $\mathcal{Y}_{\text {reg }}$, also $K_{\widehat{X}}$ is Cartier, see [32, Prop. 5.20]. So $\mathcal{R}(X)$ is numerically Gorenstein. The additions follow directly. 
In general, one cannot expect $K_{\widehat{X}}$ to be trivial, though $K_{\mathcal{Y}}$ is. An important class of varieties where this is true are the quasicones from Sect. 4.

\section{Quasicone Cox rings}

In [10], it was shown that varieties of Fano type are MDS. So in a way, the Fano type property guarantees MDS-ness, while non Fano type varieties may or may not be MDS. In this section, we give a criterion (namely being a klt quasicone) for affine varieties that guarantees MDS-ness and, moreover, guarantees preservation of MDS-ness by taking Cox rings. This is exactly what we need for iteration of Cox rings. Since we also show that Cox rings of Fano varieties are such quasicones, one can say that also the Fano type property not only guarantees MDS-ness but also its preservation.

Definition 2 An affine variety $X=\operatorname{Spec}(A)$ is called a quasicone, if one of the following equivalent conditions holds:

(i) $X$ is equipped with a $\mathbb{C}^{*}$-action and the closures of all $\mathbb{C}^{*}$-orbits meet in one common point.

(ii) $A$ is $\mathbb{Z}_{\geq 0}$-graded and $A_{0}=\mathbb{C}$.

(iii) $A$ is $\mathbb{Z}_{\geq 0}$-graded and has homogeneous generators $a_{1}, \ldots, a_{n}$ of strictly positive degree.

For a set of homogeneous generators of $A$ as in (iii) and the corresponding embedding of $X$ into $\mathbb{C}^{n}$, the origin is the common point of the orbit closures from (i), it is called the vertex of $X$. We refer to $[15,16]$ for detailed treatments of quasicones.

Lemma 1 Let $X$ be a normal quasicone. Then $\operatorname{Pic}(X)=0$.

Proof This is [35, Le. 5.1], compare also [18, Cor. 10.3] and [4, Cor. 2.14].

Remark 5 If $X$ is not normal, $\operatorname{Pic}(X)$ can be nontrivial. Consider for example $A=$ $\mathbb{C}[x, y] /\left(x^{3}-y^{2}\right)$ with $\operatorname{Pic}(A)=\mathbb{C}_{+}$.

Proposition 3 Let $X$ be an MDS that is either a quasicone or has only constant global regular functions, e.g. $X$ is complete. Then $\bar{X}$ is a quasicone. If $x$ is the vertex of $\bar{X}, \mathrm{Cl}(\bar{X}, x) \cong \mathrm{Cl}(\bar{X})$ and every irreducible component of $\operatorname{Sing}(\bar{X})$ intersects $x$ nontrivially.

Proof First assume $X$ has only constant global regular functions. We have a decomposition $H_{X}=E \times \mathbb{T}$ of the characteristic quasitorus of $X$ with nontrivial torus part $\mathbb{T}$, since otherwise $X=X_{E}$ would be quasiaffine with Proposition 1 . Then $X_{\mathbb{T}}$ has only constant global regular functions. Consider the $\mathbb{Z}^{k}$-grading of $A:=\mathcal{R}(X)$ corresponding to the quotient $\widehat{X} \rightarrow X_{\mathbb{T}}$, observe $A_{0}=\mathbb{C}$ and set $\mathfrak{m}=A \backslash A_{0}$. Since the weight cone contains no line, by appropriately projecting onto $\mathbb{Z}$, we can assume $k=1$ and $\bar{X}$ is a quasicone.

Now let $X$ be a quasicone. Here the coordinate ring $A:=\mathbb{C}[X]$ is $\mathbb{Z}_{\geq 0}$-graded and generators and relations of $A$ are of strictly positive degree. The grading of $A$ can be lifted to $\mathcal{R}(X)$ and thus $\mathcal{R}(X)$ is $\mathbb{Z}^{\operatorname{dim}(H)+1}$ graded, where $H$ is the characteristic quasitorus of $X$. As we have $\mathcal{R}(X)_{0_{H}}=A_{0}=\mathbb{C}$, the $\mathbb{Z}^{\operatorname{dim}(H)+1}$-weight cone of $\mathcal{R}(X)$ again contains no lines and by appropriately projecting onto $\mathbb{Z}$, we see that $\bar{X}$ is a quasicone.

The remaining assertions follow directly from $X$ being a quasicone.

Remark 6 If an affine MDS $X$ is a quasicone, the fan of the canonical ambient toric variety $Z$, see [5, Sec. 3.2.5], is in fact (the fan of faces of) a cone, since the torus action of $X$ comes 
from the torus action on $Z$ and thus the vertex of $X$ is the torus fixed point of $Z$. In general, the fan of the canonical ambient toric variety of affine MDS that are not quasicones is only a subfan of the fan of faces of a cone.

Proof (Proof of Theorem 2) If $X$ is projective, then the assertion follows from [20, Thm. 1.1] and Theorem 1, Proposition 3.

Now assume $X$ is a quasicone and $(X, \Delta)$ is klt. By [10, Cor. 1.4.3] with $\mathfrak{E}=\emptyset$, there is a small birational contraction $\varphi: Y \rightarrow X$, which is a log terminal model. There is a $\mathbb{Q}$-divisor $\Delta^{\prime} \geq 0$ on $Y$ such that $K_{Y}+\Delta^{\prime}=\varphi^{*}\left(K_{X}+\Delta\right)$ and $\left(Y, \Delta^{\prime}\right)$ is klt. In particular, it is $\mathbb{Q}$-factorial and $Y \rightarrow X$ is of relative Fano type [20, Le. 3.1]. Thus by [10, Cor. 1.3.2], the Cox ring of $Y$ is finitely generated over $\mathcal{O}_{X}(X)$. But since $\varphi$ is small, the Cox rings of $Y$ and $X$ coincide. So the Cox ring of $X$ is a finitely generated $\mathbb{C}$-algebra.

Since $X$ is klt, it is of globally or equivalently locally $F$-regular type, see [41, Cor. 3.4] and for the equivalence [40]. On the other hand, since $\mathcal{R}(X)$ is a Gorenstein quasicone by Theorem 1 and Proposition 3, it is of $F$-regular type if and only if it is canonical. Now we follow exactly the lines of [20, Proof of Thm. 4.7] and see that $\mathcal{R}(X)$ is canonical.

Now let $X$ be affine and assume $\bar{X}$ is a Gorenstein canonical quasicone. In particular, $\mathcal{R}(X)$ is finitely generated. By the previous discussion, we know that $X$ is klt. So we have to show that $X$ is a quasicone. But this is clear since if $T_{X} \subseteq \mathbb{T}$ is the torus part of $H_{X}$ inside the maximal torus $\mathbb{T}$ acting on $\bar{X}$, then with respect to the corresponding $\mathbb{Z}^{\operatorname{dim}\left(T_{X}\right)}$-grading of $\mathcal{R}(X)$, we have $\mathbb{C} \neq \mathbb{C}[X] \subseteq \mathcal{R}(X)_{0}$, i.e. $\operatorname{dim}\left(\mathbb{T} / T_{X}\right) \geq 0$ and $\mathbb{C}[X]_{0}=\mathbb{C}$ with respect to the corresponding $\mathbb{Z}^{\operatorname{dim}\left(\mathbb{T} / T_{X}\right)}$-grading. By projecting to $\mathbb{Z}$ as in the proof of Proposition 3 , we see that $X$ is a quasicone.

\section{Iteration of Cox rings}

Iteration of Cox rings has been introduced for varieties with a torus action of complexity one in the work [4] by Arzhantsev, Hausen, Wrobel and the author.

Definition 3 Let $X$ be an MDS. If the characteristic space $X^{(1)}:=\widehat{X}$ or equivalently the total coordinate space $\mathcal{X}^{(1)}:=\bar{X}$ is an MDS as well, we can consider their characteristic and total coordinate spaces $X^{(2)}:=\widehat{X}$ and $\mathcal{X}^{(2)}:=\overline{\bar{X}}$. By iterating this procedure, we get a commutative diagram

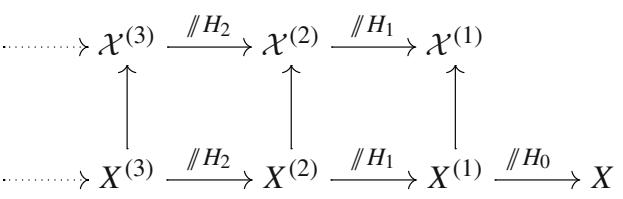

of 1-Gorenstein varieties (with the possible exception of $X$ ). Here horizontal arrows stand for quotients by characteristic quasitori and vertical arrows stand for the inclusions of the $X^{(i)}$ as open subsets with complement of codimension at least two in $\mathcal{X}^{(i)}$. We call this the iteration of Cox rings of $X$. If $X$ is affine, then so are the $X^{(i)}=\mathcal{X}^{(i)}$.

Remark 7 In the iteration of Cox rings of an MDS X, we have three possibilities:

- For each $i \in \mathbb{N}, X^{(i)}$ is an MDS with nontrivial divisor class group $\mathrm{Cl}\left(X^{(i)}\right)$. We say that $X$ has infinite iteration of Cox rings and set $N:=\infty$. 
- For some $N \in \mathbb{N}$, either $X^{(N)}$ is not an MDS or it is factorial. We call $\mathfrak{R}(X):=\mathbb{K}\left[X^{(N)}\right]$ the master Cox ring of $X$. In this case the diagram 1 is finite and we say that $X$ has finite iteration of Cox rings.

- If $X^{(N)}$ is factorial, we say that $X$ has factorial master Cox ring.

- If $X^{(N)}$ is not an MDS, we say that $X$ has non-MDS master Cox ring.

Proposition 4 For each natural number $i \leq N$, we can represent $X$ as a quotient of $X^{(i)}$ by a solvable reductive group $G_{i}$ acting freely on a subset with complement of codimension at least two and the chain of abelian quotients

$$
X^{(i)} \stackrel{/ / H_{i-1}}{\longrightarrow} \cdots \stackrel{\| / H_{2}}{\longrightarrow} X^{(2)} \stackrel{/ / H_{1}}{\longrightarrow} X^{(1)} \stackrel{/ / H_{0}}{\longrightarrow} X
$$

can be retrieved by setting $H_{j}:=G_{i}^{(j-1)} / G_{i}^{(j)}$ and $X^{(j)}:=X^{(i)} / / G_{i}^{(j-1)}$ for the $k$-th derived subgroups $G_{i}^{(k)}$ of $G_{i}$.

Proof By [4, Proof of Thm. 1.6], for each $i \leq N$, we can represent $X$ as a quotient of $X^{(i)}$ by solvable groups $G_{i}$ defined by $G_{1}:=H_{0}$ and a certain semidirect product $G_{k}:=$ $H_{k-2} \rtimes G_{k-1}$ for $k \geq 2$. Now fix some $i \leq N$ and set $\mathcal{D}_{k}:=G_{i} / G_{k}$. Then $X^{(j)}=X^{(i)} / / \mathcal{D}_{j}$. We can follow [4, Proof of Thm. 1.6], where since we do not assume $X^{(i)}$ to be factorial, we have to check that each $\mathcal{D}_{k}$-stable divisor on $X^{(i)}$ is principal in order to apply [6, Prop. 3.5]. But this holds since $X^{(i)} \rightarrow X^{(i)} / / \mathcal{D}_{k}$ factors through the characteristic spaces

$$
X^{(i)} \rightarrow X^{(i-1)} \rightarrow \ldots \rightarrow X^{(k)}=X^{(i)} / / \mathcal{D}_{k} .
$$

Thus we have that $\left[\mathcal{D}_{k}, \mathcal{D}_{k}\right]=\mathcal{D}_{k+1}$ and the assertion follows.

If $X$ has finite iteration of Cox rings, we call $G_{X}:=G_{N}$ the characteristic solvable group of $X$.

Example 1 It is clear that toric varieties trivially have finite iteration of Cox rings with polynomial master Cox ring. On the other hand, in [19], it was shown that spherical varieties have finite iteration of Cox rings with factorial master Cox ring and at most two iteration steps.

In [4], Arzhantsev, Hausen, Wrobel and the author showed that for log terminal singularities with a torus action of complexity one, Cox ring iteration is finite with factorial master Cox ring. Moreover, the possible chains for these singularities have been calculated explicitly in [4, Rem. 6.7]. In dimension two, one retrieves exactly the representation of the log-terminal singularities as finite quotients of $\mathbb{C}^{2}$, i.e. the Cox ring iteration chains of those singularities form a tree with the single root $\mathbb{C}^{2}$, see [4, Ex. 4.8]. In [4, Thm. 1.9], also the Cox ring iteration tree for compound $\mathrm{du}$ Val threefold singularities with a two-torus action has been given, and it can be seen that the Cox rings preserve the compound du Val property. Moreover, in the work [11] by Hättig and the author one can find the Cox ring iteration tree of canonical threefold singularities with a two-torus action. It turns out that all master Cox rings here are compound du Val as well.

In [27], Hausen and Wrobel gave criteria for affine varieties with a torus action of complexity one to have finite iteration of Cox rings with factorial master Cox ring. In fact, one can directly verify from their computations that all varieties with a torus action of complexity one have finite iteration of Cox rings, though with possibly non-MDS master Cox ring.

The following relative version of Cox ring iteration is the first generalization of Proposition 1, from which then directly follows the most general version, the web of Cox ring iterations from Theorem 4. 
Proposition 5 (Relative iteration of Cox rings) Let the quasitorus $H$ act on $X$, such that the good quotient $Y:=X / / H$ exists and is an almost principal $H$-bundle. Then $X$ has finite iteration of Cox rings if and only if $Y$ has so. Moreover, $X$ has factorial master Cox ring if and only if $Y$ has so and in this case, $\mathfrak{R}(X)=\mathfrak{R}(Y)$.

Proof If $H$ is torsion free, by Proposition 1 (i), $X$ and $Y$ have the same Cox ring, i.e. the same iteration of Cox rings from the second step on. So assume $H$ has torsion. From Proposition 1 (iii), we get a Cox ring iteration ladder:

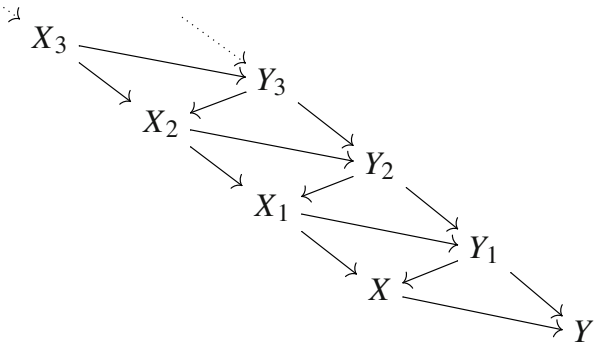

So if $X_{i}$ is an MDS, then so is $Y_{i}$ and if $Y_{j+1}$ is an MDS, then so is $X_{j}$. Thus $X$ has finite iteration of Cox rings if and only if $Y$ has so. If $\mathfrak{R}(X)$ is the factorial master Cox ring of $X$, then $X_{N}$ is an almost principal quasitorus bundle over $Y_{N}$ and $\mathfrak{R}(X)$ is factorially graded with respect to the associated character group, since it is factorial. So $\mathfrak{R}(X)$ is the Cox ring of $Y_{N}$ and thus the master Cox ring of $Y$. If on the other hand $\mathfrak{R}(Y)$ is the factorial master Cox ring of $Y$, then $Y_{N}$ is an almost principal quasitorus bundle over $X_{N-1}$ and we can apply the same argument.

Proof (Proof of Corollary 1) Let $Y \rightarrow X$ be a quotient by the solvable group $G$ as in the corollary. Then by any normal series of $G$ we get a chain of quotient presentations $Y \rightarrow$ $\cdots \rightarrow X_{3} \rightarrow X_{2} \rightarrow X$. Thus by Theorem 1, $Y$ is an MDS with factorial master Cox ring $\mathfrak{R}(X)$ and the diagram

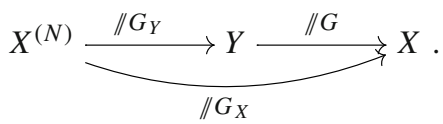

is commutative, which proves the assertion.

Now we come to the proof of our last main theorem.

Theorem 2 (Theorem 3) Let X be of Fano type or a klt quasicone. Then X has finite iteration of Cox rings with factorial master Cox ring.

Proof Let $X$ be a klt quasicone and assume it has infinite iteration of Cox rings. If $H_{i}$ is a torus for some $i \in \mathbb{N}$, then $X_{i+1}$ is factorial by [5, Prop. 1.4.1.5]. So each $H_{i}$ has a nontrivial torsion part $E_{i}$ and a possibly trivial torus part $\mathbb{T}_{i}$.

Denote $\mathfrak{X}_{1}:=X_{\mathbb{T}}=X^{(1)} / / \mathbb{T}_{0}$. By Corollary $1, X^{(2)}$ is the characteristic space of $\mathfrak{X}_{1}$. Denote by $\mathfrak{H}_{1}$ the characteristic quasitorus of $\mathfrak{X}_{1}$ and by $\mathfrak{T}_{1}$ its torus part. Define $\mathfrak{X}_{2}:=$ $X^{(2)} / / \mathfrak{T}_{1}$. By iteration of Cox rings, we get the following infinite commutative diagram, 
where all diagonal arrows are characteristic spaces:

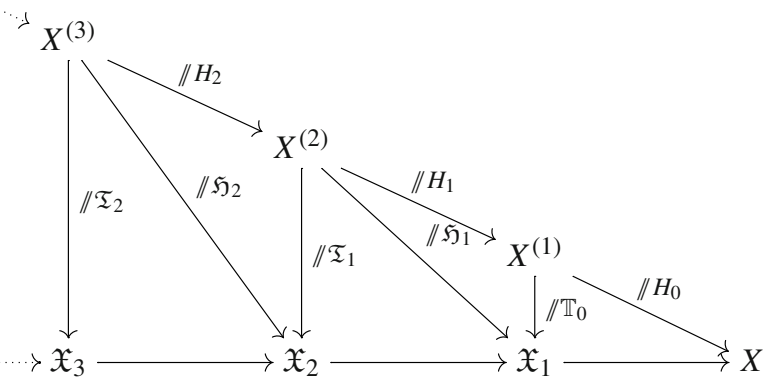

The bottom of this diagram gives a sequence of finite Galois morphisms between klt quasicones étale in codimension one such that all compositions are Galois as well, compare the lifting of the abelian group actions from Proposition 4. By [21, Thm. 1.1], all but finitely many of the $\mathfrak{X}_{i+1} \rightarrow \mathfrak{X}_{i}$ must be étale. But none of them is, since they are all ramified over the vertex, see [5, Prop. 1.6.2.5]. So $X$ must have finite iteration of Cox rings with master Cox ring $\mathfrak{R}(X)=\mathbb{C}\left[X^{(N)}\right]$ for some $N \in \mathbb{N}$.

Since all $X^{(i)}$ in the iteration of Cox rings of $X$ are Gorenstein canonical quasicones and thus MDS by Theorem 2, $X^{(N)}$ is an MDS and thus factorial. The assertion for varieties of Fano type follows directly.

Acknowledgements The author wishes to thank Giuliano Gagliardi, Helmut Hamm, Mitsuyasu Hashimoto, János Kollár, Scott Nollet, Ivo Radloff and Karl Schwede for helpful comments. In particular, he is grateful to Jürgen Hausen and Stefan Kebekus for stimulating discussions. He thanks the anonymous referee for several helpful remarks.

Funding Open Access funding enabled and organized by Projekt DEAL.

Open Access This article is licensed under a Creative Commons Attribution 4.0 International License, which permits use, sharing, adaptation, distribution and reproduction in any medium or format, as long as you give appropriate credit to the original author(s) and the source, provide a link to the Creative Commons licence, and indicate if changes were made. The images or other third party material in this article are included in the article's Creative Commons licence, unless indicated otherwise in a credit line to the material. If material is not included in the article's Creative Commons licence and your intended use is not permitted by statutory regulation or exceeds the permitted use, you will need to obtain permission directly from the copyright holder. To view a copy of this licence, visit http://creativecommons.org/licenses/by/4.0/.

\section{References}

1. Achinger, P., Ilten, N., Süss, H.: F-split and F-regular varieties with a diagonalizable group action. J. Algebra Geom. 26(4), 603-654 (2017)

2. Artebani, M., Hausen, J., Laface, A.: On Cox rings of K3 surfaces. Compos. Math. 146(4), 964-998 (2010)

3. Arzhantsev, I.: On the factoriality of Cox rings. (Russian. Russian summary) Mat. Zametki 85(5), 643-651 (2009); translation in Math. Notes 85 (2009), no. 5-6, 623-629

4. Arzhantsev, I., Braun, L., Hausen, J., Wrobel, M.: Log terminal singularities, platonic tuples and iteration of Cox rings. Eur. J. Math. 4(1), 242-312 (2018)

5. Arzhantsev, I., Derenthal, U., Hausen, J., Laface, A.: Cox rings. Cambridge Studies in Advanced Mathematics, 144. Cambridge University Press, Cambridge, viii+530 pp (2015) 
6. Arzhantsev, I., Gaifullin, S.: Cox rings, semigroups, and automorphisms of affine varieties. Sb. Math. 201(1-2), 1-21 (2010)

7. Bäker, H.: Good quotients of Mori dream spaces. Proc. Am. Math. Soc. 139(9), 3135-3139 (2011)

8. Bechtold, B.: Factorially graded rings and Cox rings. J. Algebra 369, 351-359 (2012)

9. Berchtold, F., Hausen, J.: Homogeneous coordinates for algebraic varieties. J. Algebra 266(2), 636-670 (2003)

10. Birkar, C., Cascini, P., Hacon, C.D., McKernan, J.: Existence of minimal models for varieties of log general type. J. Am. Math. Soc. 23(2), 405-468 (2010)

11. Braun, L., Hättig, D.: Canonical threefold singularities with a torus action of complexity one and $k$-empty polytopes. Rocky Mountain J. Math. 50(3), 881-939. https://doi.org/10.1216/rmj.2020.50.881

12. Brion, M.: The total coordinate ring of a wonderful variety. J. Algebra 313(1), 61-99 (2007)

13. Brown, M.: Singularities of Cox rings of Fano varieties. J. Math. Pures Appl. (9) 99(6), 655-667 (2013)

14. Cox, D.A.: The homogeneous coordinate ring of a toric variety. J. Algebra. Geom. 4(1), 17-50 (1995)

15. Demazure, M.: Anneaux gradués normaux. Introduction à la théorie des singularités, II, 35-68, Travaux en Cours, 37, Hermann, Paris (1988)

16. Dolgachev, I.: McKay correspondence. Winter 2006/07. Lecture notes, available at: www.math.lsa.umich. edu/ idolga/McKaybook.pdf

17. Elizondo, E.J., Kurano, K., Watanabe, K.-I.: The total coordinate ring of a normal projective variety. J. Algebra 276(2), 625-637 (2004)

18. Fossum, R.M.: The divisor class group of a Krull domain. Ergebnisse der Mathematik und ihrer Grenzgebiete, Band 74. Springer, New York. viii+148 pp (1973)

19. Gagliardi, G.: Luna-Vust invariants of Cox rings and skeletons of spherical varieties. arXiv:1608.08151

20. Gongyo, Y., Okawa, S., Sannai, A., Takagi, S.: Characterization of varieties of Fano type via singularities of Cox rings. J. Algebra. Geom. 24(1), 159-182 (2015)

21. Greb, D., Kebekus, S., Peternell, T.: Étale fundamental groups of Kawamata log terminal spaces, flat sheaves, and quotients of abelian varieties. Duke Math. J. 165(10), 1965-2004 (2016)

22. Hamm, H.A., Tráng, L.D.: On the Picard group for non-complete algebraic varieties. Singularités FrancoJaponaises, 71-86, Sémin. Congr., 10, Soc. Math. France, Paris (2005)

23. Hara, N., Watanabe, K.-I.: $F$-regular and $F$-pure rings vs. $\log$ terminal and log canonical singularities. J. Algebra Geom. 11(2), 363-392 (2002)

24. Hashimoto, M.: Equivariant class group. III. Almost principal fiber bundles. arXiv:1503.02133

25. Hausen, J.: Cox rings and combinatorics. II. Mosc. Math. J. 8(4), 711-757, 847 (2008)

26. Hausen, J., Süß, H.: The Cox ring of an algebraic variety with torus action. Adv. Math. 225(2), 977-1012 (2010)

27. Hausen, J., Wrobel, M.: On iteration of Cox rings. J. Pure Appl. Algebra 222(9), 2737-2745 (2018)

28. Hochster, M., Huneke, C.: Tight closure in equal characteristic zero. preprint, available at: www.math. lsa.umich.edu/ hochster/tcz.ps

29. Hu, Y., Keel, S.: Mori dream spaces and GIT. Dedicated to William Fulton on the occasion of his 60th birthday. Michigan Math. J. 48, 331-348 (2000)

30. Kawamata, Y., Okawa, S.: Mori dream spaces of Calabi-Yau type and log canonicity of Cox rings. J. Reine Angew. Math. 701, 195-203 (2015)

31. Kollár, J.: New examples of terminal and log canonical singularities. arXiv:1107.2864

32. Kollár, J., Mori, S.: Birational geometry of algebraic varieties With the collaboration of C. H. Clemens and A. Corti. Translated from the 1998 Japanese original. Cambridge Tracts in Mathematics 134, Cambridge University Press, Cambridge (1998)

33. Laface, A., Liendo, A., Moraga, J.: The fundamental group of a log terminal T-variety. Eur. J. Math. (2018). https://doi.org/10.1007/s40879-018-0296-z

34. McKernan, J.: Mori dream spaces. Jpn. J. Math. 5(1), 127-151 (2010)

35. Murthy, M.P.: Vector bundles over affine surfaces birationally equivalent to a ruled surface. Ann. Math. (2) 89, 242-253 (1969)

36. Okawa, S.: On images of Mori dream spaces. Math. Ann. 364(3-4), 1315-1342 (2016)

37. Parameswaran, A.J., van Straten, D.: Algebraizations with minimal class group. Internat. J. Math. 4(6), 989-996 (1993)

38. Saito, K.: Quasihomogene isolierte Singularitäten von Hyperflächen. (German) Invent. Math. 14, 123-142 (1971)

39. Schwede, K.: A refinement of sharply $F$-pure and strongly $F$-regular pairs. J. Commun. Algebra 2(1), 91-109 (2010)

40. Schwede, K., Smith, K.E.: Globally F-regular and log Fano varieties. Adv. Math. 224(3), 863-894 (2010)

41. Takagi, S.: An interpretation of multiplier ideals via tight closure. J. Algebra Geom. 13(2), 393-415 (2004) 
42. Xu, Ch.: Finiteness of algebraic fundamental groups. Compos. Math. 150(3), 409-414 (2014)

Publisher's Note Springer Nature remains neutral with regard to jurisdictional claims in published maps and institutional affiliations. 